\title{
Germanica
}

\section{La fiction comme mise à distance}

L'expérience de la RDA dans les narrations de Julia Schoch et d'Antje Rávic Strubel

Fiktion als Distanz. Erfahrungen mit der DDR im erzählerischen Werk von Julia Schoch und Antje Rávic Strubel

\section{Carola Hähnel-Mesnard}

\section{(2) OpenEdition}

\section{Journals}

Édition électronique

URL : http://journals.openedition.org/germanica/346

DOI : $10.4000 /$ germanica.346

ISSN : 2107-0784

\section{Éditeur}

Université de Lille

\section{Édition imprimée}

Date de publication : 1 décembre 2006

Pagination : 181-193

ISBN : 2-913857-18-3

ISSN : 0984-2632

\section{Référence électronique}

Carola Hähnel-Mesnard, "La fiction comme mise à distance », Germanica [En ligne], 39 | 2006, mis en ligne le 19 février 2010, consulté le 06 octobre 2020. URL : http://journals.openedition.org/germanica/ 346 ; DOI : https://doi.org/10.4000/germanica.346

Ce document a été généré automatiquement le 6 octobre 2020.

(C) Tous droits réservés 


\title{
La fiction comme mise à distance
}

\author{
L'expérience de la RDA dans les narrations de Julia Schoch et d'Antje \\ Rávic Strubel \\ Fiktion als Distanz. Erfahrungen mit der DDR im erzählerischen Werk von Julia \\ Schoch und Antje Rávic Strubel
}

\section{Carola Hähnel-Mesnard}

1 Dix ans après la chute du Mur et la réunification, la littérature contemporaine allemande a connu une prolifération de récits de jeunes Allemands de l'Est nés dans les années 1970 essentiellement constitués par les souvenirs d'enfance et de jeunesse d'une époque définitivement révolue et disparue avec tous ses objets tangibles. Une grande partie de ces récits fait écho à la littérature "pop » des «nouveaux archivistes » qui fixent le quotidien de leur jeunesse par l'évocation et le «catalogage» de produits, de marques et de héros de leur enfance ${ }^{1}$. À l'instar de la "génération Golf» décrite par Florian Illies, la presse a rapidement parlé d'une "génération Trabant » à propos des jeunes auteurs de l'ex-RDA ${ }^{2}$. Alors que les récits de la " génération Golf» visent à créer un sentiment générationnel fondé sur un certain esprit de vivre toujours actualisable, la même tentative à l'Est est plus problématique dans la mesure où le passé auquel se réfèrent les textes est sujet à débat et à de multiples interprétations, aussi bien au niveau individuel qu'au niveau collectif.

2 De fait, les auteurs de l'ex-RDA ont aussi la position de témoins d'une époque révolue : en dépit de leur jeunesse, ils sont nombreux à souligner qu'ils sont les «derniers Allemands de l'Est ». Cette position testimoniale, certains l'acceptent volontiers, et elle est fortement promue par les maisons d'édition, prêtes à laisser s'estomper les contrats de lecture entre fiction et document vécu. Dans ce sens, la «littérature du souvenir " des jeunes auteurs est-allemands se présente comme l'expression tout à fait contemporaine d'une évolution qui remonte aux années 1980, avec l'émergence d'un "nouvel âge de la mémoire ", phénomène qui s'est généralisé aujourd'hui avec l'importance que l'on accorde aux expressions non seulement de la mémoire collective, mais également individuelle, phénomène qui se traduit également dans la littérature. 
3 Les textes narratifs de Julia Schoch ${ }^{4}$ et Antje Rávic Strubel ${ }^{5}$ (nées en 1974) sont en décalage avec cette littérature du souvenir. Contrairement à des récits qui réclament l'authenticité du vécu et se composent d'une multitude d'anecdotes autobiographiques plaisantes, la particularité des deux auteures résulte d'une conscience accrue du caractère faillible et construit de la mémoire et du souvenir. Elle réside dans les multiples tentatives de relativiser et de tenir à distance les souvenirs, qu'ils soient personnels ou transmis par d'autres, en recourant à différents procédés de fictionnalisation. Leur vision du passé de la RDA et de la période de transition est complexe. L'expérience fondamentale est celle d'une rupture intervenue au moment de leur adolescence, à l'âge de quinze ans. Ce qui importe à Antje Rávic Strubel n'est pas le passé de la RDA, mais le fait de trouver ses repères une fois ce passé disparu. La question sous-jacente à son écriture est celle de savoir comment, aujourd'hui, on peut encore dire quelque chose sur l'Est, à quoi ressemble un être humain au passé invisible ${ }^{6}$. Selon Julia Schoch, "ce n'est pas la RDA qui manque aux Allemands de l'Ouest. C'est l'expérience d'une rupture absolue - et la chute du Mur constituait une telle rupture. C'est l'expérience fondamentale que ce qui est ne va pas de soi $»^{7}$. Les récits des deux auteures problématisent la douleur fantôme ressentie après la perte du cadre de vie habituel qui a profondément marqué les individus, tout en ayant aussi un regard sur la dimension sociale de la période de transition. Mais ils tentent d'évoquer également les conflits entre l'individu et l'État à l'époque de la RDA sans pour autant se réduire à une condamnation générale de cette période.

Dans les neufs récits de Der Körper des Salamanders (2001) de Julia Schoch, l'auteure introduit une multiplicité de points de vues narratifs afin d'éviter une lecture autobiographique. Si sa biographie se recoupe toutefois avec les expériences de certains personnages ${ }^{8}$, Julia Schoch tente d'en effacer les traces en plongeant le lecteur dans des univers fictionnels souvent énigmatiques, en élaborant des récits d'une grande intensité poétique par une narration et une écriture qui peuvent apparaître à première vue comme codées, chiffrées, cryptiques.

5 La référence au réel ne disparait pas pour autant. Dans le premier récit Le Corps de la salamandre, la narratrice à la première personne décrit soigneusement son cadre de vie et son activité : elle est barreuse d'un quatre et responsable de l'entraînement des rameuses. Quelques indices permettent de situer le récit en RDA, dans le milieu du sport de haut niveau : l'existence d'un « responsable pour l'instruction politique », vétu d'un anorak portant l'inscription «Dynamo », l'association sportive appartenant aux services de sécurité, ou encore le fait que la narratrice ne peut pas participer aux compétitions à l'Ouest ${ }^{9}$. Par ailleurs, l'esthétique du réalisme socialiste est discrètement évoquée par la description d'une peinture murale dans la cantine de l'internat (16). Ces références allusives au contexte de la RDA permettent au lecteur de situer le récit tout en laissant ouvertes des lectures multiples et en maintenant l'ambiguïté propre à la fiction. En renonçant à de plus amples descriptions aussi bien du quotidien en RDA que des expériences collectives, les récits de Julia Schoch évitent la transformation des réalités en clichés connotés par l'Ostalgie. Ce parti pris de fictionnalisation et de littérarisation situe le texte au-delà de toute prétention à la vérité testimoniale, il contribue à ce que les récits se refusent à la possibilité de devenir une interface à l'identification générationnelle.

6 La distance que l'auteure instaure par rapport à son propre vécu est thématisée de façon métafictionnelle dans «Der Exot» [ L'animal exotique »] où la narratrice, 
journaliste originaire de RDA, est invitée par la rédaction à écrire sur son enfance. Or, proposer des histoires authentiques témoignant de la "rupture dans la vie " que représente la chute du Mur, n'est pas le projet de la narratrice qui avoue que ce retour sur les lieux de son enfance suit un scénario déjà imaginé lorsqu'elle était encore enfant. Ici encore, la position de la narratrice est en décalage avec l'horizon d'attente $\mathrm{du}$ «témoignage». C'est la fiction qui paradoxalement rend les expériences de l'auteure plus vraisemblables qu'une posture testimoniale, compte tenu du jeune âge de l'auteure. Ainsi, la narratrice affirme : «Beaucoup trop tôt j'ai dû me souvenir de mon enfance, comme si cinq États étaient entre nous, et non pas un seul, comme si je pouvais me permettre de rêver d'une enfance que j'avais tout juste quittée $»^{10}$. Julia Schoch affirme ainsi une position critique quant à la possibilité de se remémorer et quant à l'exactitude des souvenirs. Car si toute mémoire est sujette à des aléas, celle de l'enfance l'est encore plus : «l'enfance est un contenu oscillant entre réalité passée (et à ce titre victime des aléas de la mémoire) et fiction », remarque Laure Himy-Pieri ${ }^{11}$.

7 Seuls deux des neufs récits du recueil se situent à l'époque de la RDA; ils mettent en scène des personnages en prise à de graves conflits existentiels, qui n'hésitent pas à recourir à des solutions radicales pour les résoudre. Si dans "Schießübung" [ Exercice de tir »], un soldat inspiré par les idées de Sartre et de Simone de Beauvoir saisit une occasion inattendue pour voler une arme et déserter, la narratrice adolescente dans Le Corps de la salamandre est en complet décalage avec son entourage. Sa vocation est l'écriture, et non pas l'aviron qu'elle pratique à l'École de sport. Pour se dérober à un entraînement physiquement pénible et monotone, elle commet régulièrement des petits actes de sabotage. Mais lorsque l'hiver trop doux pour des entraînements à l'intérieur déjoue son projet de remplir son livre bleu sur lequel flotte une Mélusine, la narratrice décide d'en finir avec cette situation. Le brouillard aidant, elle guide le quatre de façon à ce qu'il chavire: «Je pensais que la Havel pouvait également être le Styx, car nous traversions des nappes de brouillard humides vers un autre monde $»^{12}$. Cette fois, le sabotage se transforme en meurtre calculé : alors que la narratrice se dégage du bateau, les autres filles se trouvent prises en piège : «J'ai vu : le bateau avait la coque en l'air et en bas, dans l'eau, [...] les filles étaient assises dans le bateau, une image en miroir, comme si elles voulaient - une galère devenue muette sauver leur nomination dans les ténèbres ${ }^{13}$. Après cet acte de libération, la narratrice flottant sur l'eau comme une Ophélie survivante, sent émerger en elle le poème dont elle avait pour l'instant trouvé seulement le premier vers : «Laisse la salamandre [...] » $(17,28)$.

8 Ce récit est exemplaire du travail littéraire de Julia Schoch, de la transposition d'un contexte concret dans une dimension plus universelle en recourant à l'intertextualité (les motifs romantiques de la fleur bleue et de Mélusine ; Ophélie), à la mythologie, au fonds des symboles (magiques) de l'univers naturel (les animaux, les quatre éléments). En dehors de la mise à distance de l'événementiel concret d'un vécu, cette approche littéraire permet également d'intégrer des expériences spécifiquement est-allemandes dans le fonds d'expériences et de conflits humains en général dont le mythe, par exemple, est une des expressions les plus anciennes et universelles ${ }^{14}$.

9 Notons que le motif de l'eau est omniprésent dans les récits du recueil. S'il participe, comme élément originel, à la construction d'un espace référentiel universalisant de la narration, il faut également rappeler sa signification symbolique quant à la mémoire : si l'eau peut représenter l'oubli (Léthé), elle figure également comme «force 
régénératrice de la mémoire » (la fontaine de Delphes) ${ }^{15}$. Dans le récit cité, le fait que le bateau chavire équivaut à l'effacement, l'oubli volontaire, l'acte de faire table rase, d'un passé pesant. Mais ensuite, un poème jaillit au moment où la narratrice flotte sur l'eau. Ici intervient l'autre signification de l'eau comme source des poètes où, selon Assmann, "les divergences entre prophétie et souvenir s'effacent », les poètes étant inspirés par les muses, filles de mnémosyne : «Il n'y a pas de parole créatrice sans la mémoire » ${ }^{16}$. Ainsi, l'eau figure aussi bien comme élément destructeur que comme possible renouveau, se manifestant ici par l'émergence de l'écriture, mais la parole poétique tiendra inévitablement compte du passé - ce qui peut se lire comme une métaphore du projet d'écriture de l'auteure ${ }^{17}$.

10 Les deux récits traitant de l'époque de la RDA mettent en scène des personnages qui tentent de se libérer à tout prix de leur condition, qui décident de tout faire pour se réaliser eux-mêmes et accéder à une vie non déterminée par les contraintes extérieures, en l'occurrence celles imposées par l'État. Cette aspiration rappelle la conception existentialiste de la liberté - des allusions dans plusieurs récits renforcent cette hypothèse - sans toutefois tenir compte de la dimension éthique de cette pensée : la narratrice dans Le Corps de la salamandre va jusqu'au meurtre. Ce motif du meurtre sert aussi à préserver le lecteur d'une lecture autobiographique, comme c'est également le cas dans le récit «Himmelfahrt» [ Ascension»]. Celui-ci décrit la découverte par la narratrice de son père suicidé lorsque, prévenue par téléphone de sa mort imminente, elle se rend à l'appartement. La description détaillée de la pièce retarde la découverte du corps et introduit une distance objectivante et dénuée de tout pathos, distance maintenue lors de l'évocation de certains souvenirs d'enfant. On apprend enfin que la narratrice même avait poussé son père, un militaire de haut rang en RDA ne parvenant pas à s'adapter à la situation après 1989, au suicide (49). Elle voulait préserver ses souvenirs d'enfance dans lesquels la petite fille qu'elle était aurait mal supporté de voir le père retourner sa veste: "Ainsi, au moins, les situations gênantes ont-elles été épargnées à mon père. Et à moi aussi. J'aurais dû supporter de le voir à nouveau finir par s'arranger rapidement de tout. N'était-ce pas mon père qui flottait sur l'eau? $»^{18}$.

11 La transposition des expériences est-allemandes dans une dimension archaïque et parfois fantastique n'empêche pas une certaine "écriture engagée » qui tient compte de la réalité sociale. Dans «Boulevard Lipscani $n^{\circ} 3$ ", un jeune couple de chercheurs réside à Bucarest. Le jeune homme, Crohn, est à la recherche de la «formule du monde » (30), apparemment d'inspiration mallarméenne, d'une "loi du hasard» résultant d'un jeu de dés. Il impose à sa compagne de jouer aux dés plusieurs heures par jour - une vie "à contrainte », soutenue par La Disparition de Georges Perec posée sur une étagère, clin d'œil intertextuel de la pièce maitresse de l'écriture à contraintes. Aussi énigmatique que le récit puisse paraitre, il s'intéresse toutefois aux problèmes sociaux de la Roumanie de l'après-89. Il en est de même dans «Im Delta » [« Dans le delta »] où une représentante de l'Union Européenne visite en Roumanie une ancienne coopérative de pisciculture et ce qu'il en reste avant de faire un exposé sur « Le chemin éprouvant vers l'Europe » et mettre l'ancien directeur en garde contre les promesses de la liberté. C'est dans ce sens que Ingo Arendt a pu affirmer que "[1]es ruines d'une transformation ratée surgissent partout dans les récits de Julia Schoch ${ }^{19}$. Cette affirmation vaut aussi pour le roman Verabredungen mit Mattok (2004) où une marée noire sur la Baltique fournit le cadre de la rencontre de deux personnages errants qui 
dénouent peu à peu leurs liens avec la société et pour qui le passage de la frontière à l'Est devient un synonyme de libération et d'espoir.

L'expérience d'une rupture identitaire liée à un vécu en RDA et aux années de transition est présente également dans l'œuvre d'Antje Rávic Strubel. Dans un essai autobiographique publié en 2003, l'auteure développe une réflexion sur son rapport à la langue et sur ses stratégies d'écriture. L'obstacle majeur à la transposition de ses expériences en littérature est une méfiance accrue envers la langue, expérience qui remonte à son enfance. Dès l'âge de cinq ans elle avait compris le double langage de son entourage : «Passer des choses sous silence - c'est ce qui se tenait au début de mon alphabétisation $»^{20}$. Le passage à un contexte langagier différent après 1989 ne provoque pas un sentiment de libération, mais une irritation envers la parole de personnes qui «n'ont jamais été soustraites au flux du temps ", " ne se sont jamais retrouvés en dehors de la continuité des images, signes, rituels changeants ». L'écriture devient seulement possible par la construction d'un lieu intérieur d'écriture, désigné par le mot artificiel Rávic apposée à son nom. Cet artifice crée une distance qui permet la mise entre parenthèses des " attributions idéologiques ", des " exigences de réalité ", des "verdicts d'intelligibilité » et des «normes de perception " produits par la société et facilite l'articulation d'une parole propre moins infléchie par des influences extérieures.

Dans ses romans, Antje Rávic Strubel met en place de multiples stratégies narratives pour mettre à distance le vécu, insinuant ainsi un rapport équivoque au passé. Contrairement au flux rassurant des récits d'enfance de ses collègues, elle introduit des topoï propres à l'écriture postmoderne qui rendent compte de la complexité du vécu et des souvenirs et expriment la fragilité d'une existence après l'expérience d'un changement historique : la déstabilisation et la fragmentation du sujet et la perte des liens. Son premier roman offene Blende (2001) relate l'histoire d'une jeune femme, Christiane, qui quitte la RDA en 1987 pour New York où elle finit par monter un petit théâtre off à Greenwich Village. Après la chute du Mur, elle fait la connaissance d'une jeune photographe ouest-allemande, Léah, dont elle tombe amoureuse. Il s'en suit une relation conflictuelle où Léah tente de percer les secrets qui entourent Christiane. Celle-ci prend ses distances et dissimule son identité est-allemande. Elle est née en 1961 et appartient à une autre génération que l'auteure, ce qui permet à cette dernière d'introduire un décalage biographique et de signifier au lecteur le caractère fictionnel du texte. Il y a toutefois, comme chez Julia Schoch, des éléments autobiographiques dans ce roman. Si Strubel insiste sur le fait que son écriture n'est pas autobiographique, elle reconnaît en revanche qu'elle joue, après un certain temps, avec ses souvenirs et avec le matériau de sa biographie ${ }^{21}$, un jeu entre fiction et autobiographie caractéristique du roman postmoderne.

La RDA est principalement focalisée par Christiane qui a l'impression de ne rien pouvoir rapporter de très significatif de sa vie là-bas, excepté des détails insignifiants. Le particulier ne tient pas au quotidien, il n'y a pas de récit d'une dissidence héroïque à raconter, ni même d'une utopie, comme le suggère l'allusion au récit de Christa Wolf Aucun lieu. Nulle part : «Pas de légende, pas d'héroïne. Rien ${ }^{22}$. Pourtant, Christiane est profondément marquée par son passé dont témoignent particulièrement ses souvenirs d'enfance et son expérience théâtrale en RDA. Un épisode de son enfance, exposé dans le prologue, lui revient constamment: enfant, elle monte à dos d'âne le chemin qui mène à la Wartburg à Eisenach; elle y passe un week-end avec ses parents pour voir 
son oncle et sa tante ouest-allemands, et sa mère lui confie qu'il faut garder le secret autour de cette rencontre. Pour Christiane, ces images de l'enfance "n'ont pas disparu, elle ne sont pas oubliées, elles ne sont pas entrées dans un temps traversé par les décennies, dans les changements de lieu et dans les phrases toutes faites en langue étrangère. [...] Elles sont la trame qui relie ensemble tout ce qu'elle fait $»^{23}$. Les images de la Wartburg constituent un souvenir clé qui renvoie à la confrontation entre l'individu et le pouvoir et qui se recoupe ici avec des éléments de la biographie de l'auteure décrits dans son essai : c'est à partir de ce moment qu'elle prend conscience du double langage de la RDA et qu'elle développe une méfiance à son égard.

Le deuxième champ d'expérience marquant, non transposable à l'Ouest, est celui du théâtre. Lorsque Christiane monte sa première pièce à New York, elle a du mal à penser autrement qu'à partir de ses expériences est-allemandes où le théâtre servait à l'expression d'une critique voilée de la situation de la RDA. Ainsi, la narratrice introduit des réflexions sur l'utilisation de pièces antiques comme Antigone pour critiquer le pouvoir (38), sur le travail avec des gestes limités dont il fallait tirer « une multitude de significations» (127), sur les «manières de jouer discrètes, voilées» (60). Si ces expériences de pratique artistique fondent en grande partie l'identité du personnage de Christiane, ces passages font également référence à un savoir culturel, quasiment livresque, sur la fonction critique du théâtre en RDA.

Ces allusions à l'art dramatique en RDA peuvent être interprétées comme une forme d'«intertextualité substitutive» qui "signale l'impossibilité de l'écriture littéraire référentielle en même temps qu'elle la pallie $»^{24}$. En effet, les expériences du personnage ne concordent plus ici avec celles de l'auteure qui ne peut plus « rendre compte du monde en tant que tel » et "recourt à la bibliothèque ${ }^{25}$. Ce jeu avec des références culturelles atteint son paroxysme dans les douze titres des chapitres qui reprennent tous un titre de livre et un film : s'il y a des références à la photographie et à la condition postmoderne ${ }^{26}$, la plupart sont issues d'auteurs liés au contexte de la $\mathrm{RDA}$ : les romans du réalisme socialiste de Cholokhov à Ostrovski (canon scolaire), la littérature de l'exil (Leonhard Frank) et la littérature de RDA où l'auteure associe Uwe Johnson à Reiner Kunze, Christa Wolf et Maxie Wander. Il serait inutile de chercher le rapport exact à l'hypotexte, il s'agit d'un rapport d'intertextualité fortuit, sans engagement, avec tout au plus un rapport de connotation qui fait fonction de clin $\mathrm{d}^{\prime} œ \mathrm{l}^{27}$. Des références intertextuelles multiples, une des caractéristiques du roman postmoderne, semblent jalonner le texte et indiquer que le référent du roman n'est pas, dans une intention réaliste et documentaire, le réel, mais bien l'imaginaire, la dimension fictionnelle de la littérature ${ }^{28}$. Il ne s'agit pas de témoigner d'un vécu en $\mathrm{RDA}$, mais d'en présenter des possibles à travers la fiction et de le signifier au lecteur.

17 Si l'approche narrative d'Antje Rávic Strubel fait des emprunts à la boîte d'outils du roman postmoderne, c'est pour exprimer la déstabilisation du sujet après une expérience de rupture historique importante qui semble réactualiser l'impossibilité du sujet postmoderne "d'organiser son passé et son avenir dans une expérience cohérente $»^{29}$. Il apparaît que les techniques narratives ou le choix du genre signifient, à eux seuls, cette déstabilisation et cette rupture. Ainsi, la " pièce nocturne " Fremd Gehen $(2002)^{30}$ superpose plusieurs plans narratifs : le personnage principal Daniel Stillmann, étudiant en mathématiques, se trouve être lui-même le personnage d'une fiction policière inventée par le narrateur/la narratrice (cela reste flou) et son amie Marlies qui, originaire de RDA, subit les conséquences de la période de transition. Or, au cours 
du récit, ces deux plans s'entremêlent. Rávic Strubel joue ici avec les conventions de genre du roman noir qui, en tant que quête, enquête et « récit emblématique de la crise du sujet » a influencé depuis longtemps le roman contemporain dans la mesure où il permet un questionnement de l'identité et l'expression de l'instabilité de l'être ${ }^{31}$. Dans son jeu avec le genre, l'auteure s'inspire d'une œuvre emblématique de la «antidetective-novel $\aleph^{32}$ citée en exergue, la trilogie new-yorkaise de Paul Auster. Le personnage Daniel Stillmann est un clin d'œil aux deux personnages principaux de Cité de verre (1985), Daniel Quinn et Peter Stillman, et le jeu avec les plans narratifs et les personnages est une référence aux nombreuses réflexions métafictionnelles dans le roman d'Auster. Le flou entourant le narrateur à la première personne, son sexe et son éventuelle identité avec le personnage de Daniel (qui pourrait également être le meurtrier de la trame policière), inspire de la méfiance au lecteur. Le recours à ce «narrateur non fiable » souligne le jeu du récit dans sa tension entre fiction et réalité.

De façon plus évidente encore, le type du "narrateur non fiable » est repris dans le dernier roman de l'auteure, Tupolew 134 (2004), qui se base sur un fait réel: le détournement d'un avion de ligne polonais vers l'aéroport de Tempelhof à Berlin-Ouest par deux Allemands de l'Est en 1978. L'auteure s'inspire librement de ce fait divers pour imaginer le vécu en RDA de ces deux protagonistes, encore très éloignés d'elle par leur biographie. Ce faisant, elle n'essaie pas de reconstituer le passé, mais elle génère " des lacunes, des vides, des incertitudes $\aleph^{33}$. Les plans de la narration focalisent d'abord le milieu ouvrier d'une grande usine de camions à Ludwigsfelde - un topos récurrent de la littérature de RDA - où évoluent les personnages principaux. Ensuite il y a le plan du procès à Berlin-Ouest, suite au détournement de l'avion. Le dernier plan est occupé par le narrateur/la narratrice qui problématise sa propre fonction.

Dès le début, il/elle s'adresse au lecteur pour remettre en question sa capacité à reconstituer le passé et pour laisser planer le doute sur son identité ${ }^{34}$, avant de proposer un modèle d'approche de ce passé qui serait un puits (Schacht) à trois étages, reliés entre eux par des échelles. Ainsi, le narrateur livre au lecteur le mode d'emploi des chapitres qui suivent, intitulés «tout en bas » (ganz unten), « en bas» (unten), « en haut » (oben): «Vous montez et descendez en bas et tout en bas et vous montez, et sur chaque étage se tiennent ceux qui ne savent pas où aller. Des trois niveaux du temps, ils jettent des regards fixes. Ils surveillent le souvenir. L'avenir est enraciné dans le souvenir. Vous ne devriez pas me faire confiance $»^{35}$. Outre le jeu, cette fois, avec la catégorie du «narrateur non fiable » qui normalement ne s'auto-désigne pas en tant que tel ${ }^{36}$, cette interpellation du lecteur ouvre sur une réflexion concernant la possibilité et les modalités du souvenir qui, en soi, est présenté comme une catégorie instable, liée aux différentes visions du passé, du présent et même de l'avenir. Par ces incursions métafictionnelles du narrateur, l'auteure problématise son approche du passé, l'incertitude qui accompagne toutes les tentatives de le saisir et le caractère inévitablement imaginé d'une telle démarche.

Avec ce rapide parcours de l'œuvre de Julia Schoch et Antje Rávic Strubel, il a été possible de cerner une position par rapport au passé et une approche littéraire diamétralement opposées à la littérature du souvenir à prétention testimoniale d'autres auteurs de cette génération originaire de l'ex-RDA. Par différents signaux textuels ou par des réflexions métatextuelles et métafictionnelles, les textes narratifs de ces deux auteures déjouent l'idée qu'il puisse s'agir de "documents vécus ", reflétant de façon réaliste leurs expériences passées. Si expérience il y a, elle est 
toutefois limitée par leur jeune âge à l'époque des changements politiques de 1989. Elle concerne davantage (même si ce n'est pas exclusif) la rupture de 1989 que le vécu proprement dit en RDA. Ainsi, le rapport au passé pourrait se décrire pour cette génération comme une tension entre mémoire et post-mémoire, un concept proposé par Marianne Hirsch: la post-mémoire est l'expérience de ceux qui n'ont pas directement vécu des événements, mais qui ont été dominés par les récits de la génération précédente, directement concernée. Ce qui caractérise la post-mémoire, c'est que le rapport à son objet n'est pas établi par le souvenir, mais par un investissement imaginatif et créatif ${ }^{37}$. Pour exprimer ce rapport fluctuant et incertain au passé, Julia Schoch et Antje Rávic Strubel inscrivent leurs expériences dans la création littéraire et leurs textes ne laissent pas de doute sur le caractère imaginaire et fictionnel. Toutefois, les expériences qu'elles portent en elles - et qui font qu'elles ne sont pas une véritable "seconde génération" - ont une influence certaine sur leur vision du monde actuel et font de l'Est, comme l'exprime Julia Schoch, « un principe », «[...] où, avec la somme de mes expériences (imaginaires), je puise des questions et des problèmes que je discute pour le présent ", tout en précisant qu'il revient à l'art de les exprimer $^{38}$.

\section{NOTES}

1. Cf. Moritz Baßler : Der deutsche Pop-Roman. Die neuen Archivisten, Munich, Beck, 2002.

2. Cf. Susanne Leinemann/Antje Schmelcher: «Generation Trabant. Angekommen im neuen Deutschland ? "Zonenkinder" im Gespräch », in Die Welt, 9 novembre 2002.

3. Cf. Régine Robin : La mémoire saturée, Paris, Stock, 2003, p. 17.

4. Julia Schoch a grandi à Eggesin près de la frontière polonaise, importante ville de garnison en RDA où son père était officier. De 1992 à 1999 elle a fait des études de germanistique et romanistique à Potsdam, Paris et Bucarest. Elle a ensuite enseigné à l'Institut d'Études Romanes de l'Université de Potsdam. Après son recueil Der Körper des Salamanders (2001), très favorablement accueilli par la critique, elle publie le roman Verabredungen mit Mattok (2004). En 2005, elle reçoit le prix du jury associé au prix Ingeborg Bachmann.

5. Antje Strubel a passé son enfance et sa jeunesse à Ludwigsfelde avant de suivre une formation de libraire à Berlin. Elle a fait des études de littérature américaine, de germanistique et de psychologie à Potsdam et New York où elle a aussi travaillé dans un théâtre. Cette expérience new-yorkaise se traduit dans son premier roman offene Blende (2001). Après le roman en épisodes Unter Schnee (2001) et le récit Fremd gehen (2002), elle a publié en 2004 son deuxième grand roman Tupolew 134 qui lui a valu en 2005 le prix littéraire de la ville de Marburg et le prix pour jeunes auteurs attribué en même temps que le prix littéraire de Brême.

6. Cf. Susanne Messmer : « Wie durch einen Filter », in TAZ, 24 juillet 2001.

7. Cf. Susanne Leinemann/Antje Schmelcher : «Generation Trabant... », op. cit.

8. Cf. «Lust an der Kunst. Heute vorgestellt: Julia Schoch», in Portal. Die Potsdamer Universitätszeitung, décembre 2001.

9. Julia Schoch : Der Körper des Salamanders (2001), Munich/Zurich, Piper, 2002, p. 15, 19. Par la suite, les références à ce recueil se trouvent entre parenthèses. 
10. «Viel zu früh war es, dass ich mich an meine Kindheit erinnern musste, als lägen fünf Staaten und nicht nur einer zwischen uns, als könnte ich es mir leisten, von einer Kindheit zu träumen, der ich doch gerade erst entkommen war. » Ibid., p. 111.

11. Laure Himy-Pieri : "Histoire d'enfances, histoire de l'enfance", in Anne Chevalier/Carole Dornier (éds.) : Le Récit d'enfance et ses modèles, Actes du colloque de Cerisy (27/09 - 01/10 2001), Presses universitaires de Caen, 2003, pp. 105-116, ici : p. 105.

12. «Ich dachte, daß die Havel auch der Styx sein konnte, denn wir durchquerten feuchte Nebelfelder in eine andere Welt. " Julia Schoch, Der Körper des Salamanders, op. cit., p. 23.

13. «Ich sah: Das Boot lag mit dem Rumpf nach oben, und unten im Wasser, unter der Nebelwand, saßen die Mädchen im Boot wie ein Spiegelbild, als wollten sie - eine stumm gewordene Galeere - ihre Berufung in die Unterwelt retten. » Ibid., p. 27 sq.

14. Voir à ce sujet Franz Fühmann pour qui le mythe «[...] donne la possibilité de mesurer l'expérience individuelle, avec laquelle on serait à nouveau seul, à des modèles de l'expérience de l'humanité ». Cf. "Das mythische Element in der Literatur» (1974), in id.: Essays, Gespräche, Aufsätze 1964-1981, Rostock, Hinstorff Verlag, 1983, p. 96.

15. Cf. Aleida Assmann : Erinnerungsräume. Formen und Wandlungen des kulturellen Gedächtnisses (1999), Munich, Beck, 2003, p. 171.

16. Ibid.

17. Une autre interprétation serait de considérer l'eau comme symbole du passage, du changement, comme chiffre de la transformation politique. Cf. Anne Fleig: "Osten als Himmelsrichtung. Grenzübergänge » in Julia Schochs Erzählung Der Körper des Salamanders, in Christiane Caemmerer, Walter Delabar, Helga Meise (éds.) : Fräuleinwunder literarisch. Literatur von Frauen zu Beginn des 21. Jahrhunderts, Francfort/Main, Peter Lang, 2005, pp. 175-190, ici p. 184.

18. "So sind die Peinlichkeiten dem Vater wenigstens erspart geblieben. Und mir auch. Daß ich hätte mitansehen müssen, wie er schnell wieder mit allem zurechtgekommen wäre. Schwamm da nicht der Vater auf dem Wasser? "Julia Schoch : Der Körper des Salamanders, op. cit., p. 44.

19. Ingo Arendt : «Riss. Eleganz und Härte », in Freitag, 22 novembre 2002.

20. Antje Rávic Strubel : "X - Was ist das und kann man es noch benutzen? ", in EdiT n³1, avril 2003, disponible sur le site www.antjestrubel.de. (et citations suivantes).

21. Cf. «Schreiben für den Briefkasten. In weiter Ferne so nah: Die Ernst-Willner-Preisträgerin Antje Rávic Strubel... », in Berliner Morgenpost, 23 juillet 2001.

22. «Aber tatsächlich gab es nichts, was sie ihm sagen konnte, nichts, außer ein paar Ungereimtheiten (Männer mit grauen Dederon-Socken in Römersandalen), den Pflichtimpfungen gegen Kinderkrankheiten [...]. Dazu kam noch die Erleichterung und ein Punkt der Scham, weil es nichts zu erzählen gab. Keine Legende, keine Heldin. Nichts. » Antje Rávic Strubel : Offene Blende (2001), Munich, dtv, 2003, p. 54.

23. «Sie kommen wieder, die Bilder der Kindheit. Sie sind nicht verschollen, vergessen, eingegangen in die laufende Zeit von Jahrzehnten, in Ortsveränderungen und fremdsprachige Floskeln. [...] Sie sind das Gerüst, das alles, was sie tut, zusammenhält. » Ibid., p. 242 sq.

24. Cf. Tiphaine Samoyault : L'intertextualité. Mémoire de la littérature, Paris, Nathan, 2004, p. 85.

25. Ibid.

26. Sont cités Sur la photographie de Susan Sontag, La chambre claire de Roland Barthes, le film Blow-up d'Antonioni et Esthétique de la disparition de Paul Virilio.

27. Ceci vaut également pour la référence à Jahrestage de Uwe Johnson dans le premier chapitre, bien que certains critiques littéraires aient voulu retrouver le ton de Johnson chez la jeune auteure.

28. Le roman postmoderne a "problématisé les certitudes du réalisme littéraire", en introduisant l'expérience de la discontinuité à travers le fragmentaire, le superficiel et une multitude de choses et de signes (à laquelle on peut inclure la référence intertextuelle). Cf. Heinz Ickstadt: «Die unstabile Postmoderne oder: Wie postmodern ist der zeitgenössische 
amerikanische Roman?", in Klaus W.Hempfer (éd.): Poststrukturalismus - Dekonstruktion Postmoderne, Stuttgart, Franz Steiner Verlag, 1992, pp. 39-51, ici p. 42. Dans Offene Blende (p. 87), le rapport au réalisme est évoqué sous l'angle de la photographie : dans un dialogue entre Léah et Christiane, cette dernière, socialisée en RDA, affirme une position proche de celle, "réaliste ", « analogique », de Barthes (La chambre claire, Paris, Gallimard, Seuil, 1980, p. 138), alors que Léah insiste sur le caractère artificiel et manipulable de la photo.

29. Cf. Frederic Jameson: "Postmoderne - zur Logik der Kultur im Spätkapitalismus », in Andreas Huyssen/Klaus R. Scherpe (éds.) : Postmoderne. Zeichen eines kulturellen Wandels, Reinbek, Rowohlt, 1986, pp. 45-102, ici : p. 70.

30. Antje Rávic Strubel : Fremd Gehen. Nachtstück, Munich, dtv, 2002.

31. Cf. Jacques Dubois : Le roman policier ou la modernité (1992), Paris, Armand Colin, 2005, p. 65.

32. Cf. Carsten Springer: Crises: The Works of Paul Auster, New York, Peter Lang, 2001, p. 107sq.

33. Cf. Lothar Müller : "Etwas geht immer schief. Laudatio auf die Förderpreisträgerin Antje Rávic Strubel », Bremer Literaturpreis 2005, www.literarische-woche.de/2005/r_mueller.htm, p. 2.

34. Antje Rávic Strubel : Tupolew 134, Munich, Beck, 2004. À propos du personnage de Katja Siems, on apprend : «Ihre Geschichte ist wahr. Aber wie wollen Sie Wahrheit beweisen. » (p. 10) et «Sie werden irgendwann fragen : Wer spricht da. [...] Sie glauben, ich wäre Katja. » (p. 11).

35. "Sie gehen hoch und runter und ganz runter und hoch, und auf jeder Etage stehen die, die nicht wissen, wo sie hin sollen. Sie starren aus allen drei Ebenen der Zeit. Sie überwachen die Erinnerung. Die Zukunft ist eine Wurzel aus der Erinnerung. Sie sollten mir nicht vertrauen. » (ibid., p. 12).

36. Si l'interpellation du lecteur et les prises de positions personnelles sont caractéristiques d'un «narrateur non fiable» (« unreliable narrator»), il ne se démasque pas de façon volontaire comme c'est la cas ici, dès le début du roman. Cf. Ansgar Nünning: «Unreliable Narration zur Einführung: Grundzüge einer kognitiv-narratologischen Theorie und Analyse unglaubwürdigen Erzählens ", in id. (éd.): Unreliable Narration. Studien zur Theorie und Praxis unglaubwürdigen Erzählens in der englischsprachigen Erzählliteratur, Trier, Wissenschaftlicher Verlag, 1998, pp. 3-39, ici p. 6.

37. Hirsch a développé ce concept pour la seconde génération des survivants de l'Holocauste, mais suggère son application possible à d'autres événements et expériences culturelles et collectives traumatisantes. Cf. Marianne Hirsch: Family Frames. Photography, Narrative and Postmemory, Cambridge, Harvard University Press, 1997, p. 22.

38. Cf. l'intervention de Julia Schoch au colloque pOst-West: http://www.uni-lueneburg.de/fb3/ postwest/pr_postwest7.htm

\section{RÉSUMÉS}

Dans le paysage actuel de la littérature du souvenir des auteurs est-allemands nés dans les années 1970, les œuvres de Julia Schoch et d'Antje Ravic Strubel font exception. Contrairement aux autres écrivains dont les récits s'apparentent à des documents de témoins d'une époque, ces deux auteures ne se livrent pas à une reconstitution fidèle de leur enfance. Au contraire, elles sont conscientes du caractère faillible et lacunaire du souvenir. Si des expériences vécues en RDA ne sont pas absentes des récits, elles sont toutefois présentées de façon distanciée en introduisant 
d'une façon ou d'une autre des éléments de fiction. Lorsque dans ses récits, Julia Schoch convoque la mythologie, des symboles ou des éléments naturels et animaliers comme l'eau, elle le fait pour ancrer dans un contexte universel des expériences vécues à l'époque de la RDA et de la période de transition. Chez Ravic Strubel, les techniques narratives caractéristiques du roman postmoderne reflètent sa propre rupture biographique : intrigue policière, jeu sur la perspective et les différents plans de la narration, utilisation de narrateurs improbables. Un autre indice du recours à la fiction est la présence de nombreuses traces intertextuelles qui signifient que le référent du récit n'est pas la réalité (ou le vécu), mais la littérature avec toutes ses capacités de création et d'imagination de vécus possibles.

In der aktuellen Erinnerungsliteratur ostdeutscher Autoren, die in den 1970er Jahren geboren wurden, bilden die Werke von Julia Schoch und Antje Rávic Strubel eine Ausnahme. Beide Autorinnen interessieren sich nicht wie andere für die quasi-authentische Rekonstruktion ihrer Kindheit in Lebensberichten, die an Dokumente von Zeitzeugen erinnern. Im Gegenteil sind sie sich des fehlbaren Charakters von Erinnerung sowie deren Leerstellen bewusst. Zwar beinhalten ihre Erzählungen und Romane Erlebnisse und Erfahrungen aus der DDR, doch werden diese mittels verschiedener Fiktionalitätsstrategien distanziert dargestellt. In Schochs Erzählungen werden Mythologie, Natur- und Tiersymbole bzw. Elemente wie das Wasser aufgerufen, um genuin ostdeutsche Erfahrungen, die sowohl die DDR als auch die Übergangsperiode betreffen, in einem universellen Kontext zu verankern. Der biographische Bruch der Wende findet bei Rávic Strubel in den (postmodernen) Erzähltechniken selbst seinen Ausdruck: Rückgriff auf Elemente des Kriminalromans, Spiel mit der Erzählperspektive und den verschiedenen Erzählebenen, Einsatz unglaubwürdiger Erzähler. Ein weiteres Fiktionalitätsindiz sind die zahlreichen intertextuellen Verweise, die andeuten, dass der Referent des Erzählten nicht die Realität (oder das Erlebte) ist, sondern die Literatur mit ihren Fähigkeiten zur Schaffung und Imagination verschiedener möglicher Vergangenheiten.

\section{INDEX}

Mots-clés : RDA

oeuvrecitee Der Körper des Salamanders, Verabredungen mit Mattok, Offenen Blende, Unter Schnee, Fremd gehen, Tupolew 134

\section{AUTEURS}

\section{CAROLA HÄHNEL-MESNARD}

École Polytechnique 